\title{
Precision Treatments in Epilepsy
}

Scott Demarest ${ }^{1}\left[{ }^{\mathbb{D}} \cdot\right.$ Amy Brooks-Kayal $^{2}$

Accepted: 17 October 2021 / Published online: 26 October 2021

(c) The American Society for Experimental NeuroTherapeutics, Inc. 2021
The genomic revolution has resulted in the discovery of hundreds of genetic etiologies for a variety of epilepsies and allowed science to probe the mechanisms of acquired epilepsies for novel understanding of the pathophysiology behind epilepsy broadly. Many of genetic epilepsy syndromes are associated with abnormal development and collectively are referred to as developmental and epileptic encephalopathies (DEE) [1]. Individual genetic etiologies are rare, but collectively the DEEs represent $10 \%$ of all childhood epilepsies and account for a disproportionate burden of disability and mortality. Over the last decade, we entered the second phase of the genomic revolution where our increasing understanding of genomic mechanisms is being used for the development of novel therapies, and we entered into the era of precision therapeutics.

Novel therapeutics targeting the hundreds of newly identified genetic etiologies for epilepsy has also exposed important deficits in our knowledge of these epilepsies. The severity and course of epilepsy and other comorbid conditions across the lifespan and how best to measure these symptoms is not well established for many genetic epilepsies. Particularly for the DEEs, there is increasing recognition that novel therapeutics that target the fundamental pathogenic mechanism should ideally improve more symptoms than just seizures. In many cases, improvement in developmental domains, movement disorders, cortical visual impairment, or other comorbidities are often as important to families as reducing the frequency and severity of seizures. Drs. Palmer, Howell, and Scheffer discuss how natural history studies lay critical groundwork toward clinical trial readiness, particularly for disease-modifying trials, by defining the severity and change in these symptoms overtime [2]. High-quality

Scott Demarest

Scott.Demarest@childrenscolorado.org

1 Department of Pediatrics and Neurology, University of Colorado School of Medicine and Children's Hospital Colorado, Aurora, CO, USA

2 Department of Neurology, University of California Davis School of Medicine, Sacramento, CA, USA natural history studies inform clinical trial design by assessing how outcome measures perform in a specific population and by identifying subgroups of patients within a particular genetic etiology that have different phenotypes and pathophysiology and may not be appropriate to be in the same clinical trial, for example, in SCN2A-related DEE where there are both loss of function and gain of function variants.

There is increasing recognition that the standard 3 -month randomized control trial is not appropriate for disease-modifying trials. A combination of novel outcome measures and new clinical trial designs will increasingly be required to demonstrate the benefits of these precision treatments. Drs. Brock, Demarest, and Benke have outlined many of the challenges and potential solutions [3]. Novel disease-modifying treatments may necessitate alterations in the duration and interval of measures in trials and the resulting burden on families, create difficulty blinding some novel treatments, and raise a variety of complicated ethical questions in this shifting treatment and clinical trial paradigm. Outcome measures may typically include traditional epilepsy frequency assessments but should also seek to assess the impact of the epilepsy on daily living and the developmental and other comorbid features seen in patients with DEEs. In many cases, the primary outcome measure may not be epilepsy related.

An ideal supplement to any of these novel clinical trial designs would be a reliable and accessible biomarker. Thus far, the search for high-quality tissue biomarkers for genetic epilepsies has yielded few concrete results, but many functional biomarkers, like quantitative EEG and evoked potentials, show promise. Drs Armstrong and Marsh discuss the likely utility of these biomarkers in genetic epilepsies, but also caution that important work remains to ensure that we understand the strengths and weakness of these biomarkers for genetic epilepsies [4].

The development of novel therapies for several genetic epilepsies has also ignited increased interest in accurate and timely recognition of patients with potentially treatable conditions. While our rate of gene discovery has been exponential over the last two decades, many patients with presumed 
genetic etiologies remain undiagnosed, and significant questions remain around equitable access to genetic testing in developed countries and even more so in developing countries. Drs. Chen and Mefford's update of current testing strategies including karyotypes, chromosomal microarray, panels, and whole exome or genome as well as the current limitation of each is critical to our accurate interpretation and use of these tests clinically [5]. Clinicians must understand what types of genetic epilepsies we may still be missing, including somatic variants isolated to the brain, ring chromosome change, recently discovered epilepsy associated genes, and intronic variants disrupting gene processing based on the type of test we choose to order.

Our ability to elucidate the underlying pathogenic mechanisms of genetic epilepsies has not been able to keep pace with the rapid progress in gene discovery spurred by nextgeneration sequencing technology over the last two decades. However, recently developed screening platforms, zebrafish models, induced neurons, and organoids, are improving the efficiency of these explorations and have advantages over traditional model system in that they are derived from patients (in the case of iPSCs) and therefore genetically identical. Drs. Shcheglovitov and Peterson have provided an excellent overview of these advantages and remaining challenges with numerous examples [6]. These platforms are increasingly being utilized for drug screening as well as to understand the basic pathogenic mechanisms of disease, which is critical to the development of novel therapies. Additionally, they point out that induced neuron models still struggle to generate fast-spiking interneurons which are important when looking at the network level impact of a genetic variant.

These screening platforms as well as traditional models have been particularly important in the pursuit of improved small molecule treatments for genetic epilepsies as discussed by Dr. Goldberg. He points out that there has been a great deal of interest both in repurposing and novel small molecules for genetic epilepsies particularly for channelopathies (SCN1A, SCN2A, SCN8A, KCNQ2/3, KCNT1) which have numerous existing drugs that may interact with a channel. However, Dr. Goldberg warns us that it is important to understand not only the impact of that variant on its corresponding protein product but also the cellular and network level impact of that alteration and points to the example of SCN1A that results in a loss of function of the NAV1.1 but hyperexcitability at the network level. Additionally, there may be a need to assess function at an individual variant level in some cases as there can be both gain of function or loss of function in the same gene, as seen in SCN2A and SCN8A [7].

This is a theme echoed by Drs. Carvill, Tyler, Hesselberth, and Demarest [8] in their discussion of novel therapies that are focused on correcting the genes themselves, the processing of genes, or delivery of the final protein product. They point out that there are strengths and limitations to each of these therapeutic approaches that need to be rationally paired to the mechanism of disease associated with a particular gene and variant leading to epilepsy. Gain of function or dominant negative mechanisms, for example, may be unlikely to benefit from whole gene replacement but could benefit from a knock down antisense oligonucleotide (ASO). Similarly, haploinsufficiency mechanisms that necessitate an increase in the final protein product may benefit from gene replacement, protein replacement, or some ASO strategies. However, some genes may be more tolerant of over expression than others, and this may influence which therapeutic choice is pursued for a particular condition. Gene editing promises to solve many of these problems by correcting the variant defect as discussed by Drs. Carpenter and Lignani [9]. Gene editing technologies, mostly based on CRISP/Cas systems, show the potential to precisely alter a variety of types of variants which could be deployed to directly correct a specific recurrent variant or to influence gene regulators including $\mathrm{X}$ inactivation. These approaches may allow intrinsic regulatory processes to be utilized in some cases; however, significant challenges remain related to which types of corrections can be made and with what fidelity as well as the vector technology required to access sufficient cells similar to gene replacement approaches.

Important advancements have been made in Dravet syndrome and Angelman syndrome with multiple novel therapies in the clinical trial phase for these conditions. This provides a critical opportunity to understand how these various approaches are being applied to alter the treatment paradigms of genetic epilepsies. Drs. Isom and Knupp discuss the promising novel antisense oligonucleotide and adeno-associated virus approaches to treat Dravet syndrome but also point out the numerous challenges raised by these potential therapies including the potential need to screen novel SCN1A variants to ensure the correct therapeutic approach, a lack of reliable, valid outcome measures, and elucidation of a roadmap to determining which patients will benefit the most from which treatment [10]. Drs. Copping, McTighe, Fink, and Silverman discuss Angelman syndrome animal models and the small molecule and other novel therapeutic approaches that are currently in the pipeline as potential disease-modifying treatments for Angelman syndrome [11]. A particularly clever strategy is blocking or disrupting the UBE3A non-coding RNA antisense transcript that turns off the paternal (presumably normal) copy, thereby allowing expression of the $U B E 3 A$ gene.

Brain malformations are now recognized to frequently result from both mosaic (somatic) and non-mosaic (germline) genetic variants in numerous genes, particularly within the mTOR pathway. The structural changes resulting from these variants present both challenges and opportunities as discussed by D'Gama and Poduri [12]. Early successes in tuberous sclerosis with both vigabatrin and 
everolimus are encouraging when considering targeted treatments for mTORopathies that are not amenable to or fully treated by surgical interventions. Ongoing development of new mTOR inhibitors as well as genomic therapies shed light on solving ongoing challenges around identification of variants (particularly somatic ones) as well as better understanding the impact of specific variants.

Genetic testing for epilepsy provides the highest yield in individuals with neonatal onset epilepsy, and as such the results of genetic testing can have a profound impact on the care of neonate [13]. This, combined with the unique physiology of neonatal brains, requires precise treatment approaches for neonates that may be different than at other ages, as discussed by Drs. Ziobro, Eschbach, and Shellhaus [14]. Several novel epilepsy treatments including potassium channel openers and tyrosine receptor kinase B modulators may offer alternatives to traditional anti-seizure medications that are more appropriate to the neonatal brain physiology. Additionally, many known treatable genetic and metabolic epilepsies have seizure onset in the first years of life. Strategies for early identification and appropriate treatment of these infants are essential.

Finally, exciting scientific advances may allow us to prevent epilepsy before it starts. Post-traumatic epilepsy now has numerous animal models, yielding improved identification of changes in gene expression and inflammatory responses that occur as part of epileptogenesis after brain injury. Once identified, it is critical to determine what changes appear to be adaptive vs maladaptive in order to most effectively design novel therapies to prevent epileptogenesis, a topic explored by Drs. Dulla and Pitkänen [15]. They also evaluate the large body of preclinical work targeting various epileptogenic cascades, as well as the development of biomarkers to accurately identify those patients at highest risk of PTE.

Advancements in genomic technology have led to a therapeutic revolution in epilepsy and other neurologic conditions, which holds incredible potential to treat the previously untreatable. However, it is critical to have ongoing assessment of our collective knowledge. We must understand the limitations and potential risk of these new therapies to allow treating physicians to navigate complicated risk benefit discussions with families and patients considering these treatments.
Supplementary Information The online version contains supplementary material available at https://doi.org/10.1007/s13311-021-01147-x.

Required Author Forms Disclosure forms provided by the authors are available with the online version of this article.

\section{References}

1. Scheffer IE, Berkovic S, Capovilla G, et al. ILAE classification of the epilepsies: Position paper of the ILAE Commission for Classification and Terminology. Epilepsia. 2017;58(4):512-521.

2. Palmer, Howell, Scheffer IE. Natural History Studies and Clinical Trial Readiness for Genetic Developmental and Epileptic Encephalopathies. Neurotherapeutics. 2021.

3. Brock DC, Demarest S, Benke TA. Clinical Trial Design for Disease-Modifying Therapies for Genetic Epilepsies. Neurotherapeutics. 2021.

4. Armstrong C, Marsh ED. Electrophysiological Biomarkers in Genetic Epilepsies. Neurotherapeutics. 2021.

5. Chen WL, Mefford HC. Diagnostic Considerations in the EpilepsiesTesting Strategies, Test Type Advantages, and Limitations. Neurotherapeutics. 2021.

6. Shcheglovitov A, Peterson RT. Screening Platforms for Genetic Epilepsies-Zebrafish, iPSC-Derived Neurons, and Organoids. Neurotherapeutics. 2021.

7. Goldberg EM. Rational Small Molecule Treatment for Genetic Epilepsies. Neurotherapeutics. 2021.

8. Carvill, G. L., T. Matheny, J. Hesselberth and S. Demarest. Haploinsufficiency, Dominant Negative, and Gain-of-Function Mechanisms in Epilepsy: Matching Therapeutic Approach to the Pathophysiology. Neurotherapeutics. 2021.

9. Carpenter JC, Lignani G. Gene Editing and Modulation: the Holy Grail for the Genetic Epilepsies? Neurotherapeutics. 2021.

10. Isom LL, Knupp KG. Dravet Syndrome: Novel Approaches for the Most Common Genetic Epilepsy. Neurotherapeutics. 2021.

11. Copping NA, McTighe SM, Fink KD, Silverman JL. Emerging Gene and Small Molecule Therapies for the Neurodevelopmental Disorder Angelman Syndrome. Neurotherapeutics. 2021.

12. D'Gama AM, Poduri A. Precision Therapy for Epilepsy Related to Brain Malformations. Neurotherapeutics. 2021.

13. Truty R, Patil N, Sankar R, et al. Possible precision medicine implications from genetic testing using combined detection of sequence and intragenic copy number variants in a large cohort with childhood epilepsy. Epilepsia open. 2019;4(3):397-408.

14. Ziobro JM, Eschbach K, Shellhaas RA. Novel Therapeutics for Neonatal Seizures. Neurotherapeutics. 2021.

15. Dulla CG, Pitkänen A. Novel Approaches to Prevent Epileptogenesis After Traumatic Brain Injury. Neurotherapeutics. 2021.

Publisher's Note Springer Nature remains neutral with regard to jurisdictional claims in published maps and institutional affiliations. 